Research Article

\title{
The Stability of Certain Motion of a Charged Gyrostat in Newtonian Force Field
}

\author{
A. A. Elmandouh (D) $^{1,2}$ and Fatimah H. Alsaad ${ }^{1}$ \\ ${ }^{1}$ Department of Mathematics and Statistics, College of Science, King Faisal University, P.O. Box 400, \\ Al-Ahsaa 31982, Saudi Arabia \\ ${ }^{2}$ Department of Mathematics, Faculty of Science, Mansoura University, Mansoura 35516, Egypt \\ Correspondence should be addressed to A. A. Elmandouh; aelmandouh@kfu.edu.sa
}

Received 7 November 2020; Revised 1 December 2020; Accepted 4 February 2021; Published 22 February 2021

Academic Editor: Elbaz Abouelmagd

Copyright (c) 2021 A. A. Elmandouh and Fatimah H. Alsaad. This is an open access article distributed under the Creative Commons Attribution License, which permits unrestricted use, distribution, and reproduction in any medium, provided the original work is properly cited.

\begin{abstract}
This work aims to study the stability of certain motions of a rigid body rotating about its fixed point and carrying a rotor that rotates with constant angular velocity about an axis parallel to one of the principal axes. This motion is presumed to take place due to the combined influence of the magnetic field and the Newtonian force field. The equations of motion are deduced, and moreover, they are expressed as a Lie-Poisson Hamilton system. The permanent rotations are calculated and interpreted mechanically. The sufficient conditions for instability are presented employing the linear approximation method. The energyCasimir method is applied to gain sufficient conditions for stability. The regions of linear stability and Lyapunov stability are illustrated graphically for certain values of the parameters.
\end{abstract}

\section{Introduction}

A gyrostat is a simple multibody which consists of a rigid body and other bodies which are usually called rotors moving in such a way that their motion does not change the distribution of mass for the gyrostat [1]. The gyrostat is also well known in the literature as a dual-spin body due to the motion of the two bodies which compose the gyrostat. Volterra [2] had first introduced the notions of gyrostat when he strived to study the motion of the Earth's polar axis and interpret variations in the Earth's latitude by means of the internal motion which keeps the mass distribution of the planet fixed. This model is used in a variety of numerous applications in different branches of physics besides their classical applications in astronomy and mechanics. For example, the gyrostat was utilized as a model of the Earth that takes into account some stationary transport processes on it [2], as a model of the atmosphere and of rotating fluid (e.g., [3]) and as a controlling device in satellite dynamics (e.g., [4]).
Most of the works related to the rigid body and its extension to gyrostat can be assorted into three categories. The first is the integrability problem and the searching for the complete set of the first integrals of the motions. Borisov and Mamaev [5] contain most of those integrable problems up to 2001, and some cases were presented by several authors (see, e.g., [6-10]). The second category regards the problem of study periodic solutions, bifurcation, and chaos in some problems of rigid body-gyrostat (see, e.g., [11-14]). The third one is the stability problem of the equilibria in the dynamics of a rigid body-gyrostat moving in an orbit or about its fixed point (see, e.g., [15-23]).

The current work is interested in studying the stability of permanent rotations for the motion of a charged gyrostat moving due to the combined influence of the magnetic field and Newtonian force field. This work is regarded as an extension of some previous works. In [17], Iñarrea et al. examined the stability of permanent rotations of a heavy rigid body carrying a rotor that rotates about one of the principal axes by a constant angular velocity. This study was 
followed by Elmandouh who studied this problem in the case of a charged heavy gyrostat [20]. Vera studied the stability of relative equilibrium for a gyrostat in Newtonian force field [19].

This work is organized as follows: in Section 2, we deduce equations of the motion and rewrite them as a Lie-Poisson Hamilton system. Section 3 contains the permanent rotations and their interpretation mechanically. In Section 4, we study the stability of those permanent rotations by applying both methods linear approximation method and energyCasimir method. Section 5 involves the results found.

\section{Equations of Motion}

We consider the rotation of a charged rigid body about its fixed point and assume this body carries an axisymmetric rotor aligning along one of the principal axes of the body and rotating with a constant angular velocity. This motion is assumed to happen due to the combined influence of a homogeneous magnetic field $\overrightarrow{\mathscr{H}}$ and Newtonian forces field. For motion description, we choose two frames $O \xi_{1} \zeta_{1} \eta_{1}$ and $O \xi_{2} \zeta_{2} \eta_{2}$ fixed in the space and in the body, respectively (see Figure 1). Furthermore, the body frame $O \xi_{2} \zeta_{2} \eta_{2}$ is assumed to be the principal axes of the inertia at the fixed point $O$, and consequently, the principal inertia matrix of the gyrostat is $\square=\operatorname{diag}(A, B, C)$. Let $\vec{\gamma}=\left(\gamma_{1}, \gamma_{2}, \gamma_{3}\right)$ and $\vec{\omega}=\left(\omega_{1}, \omega_{2}, \omega_{3}\right)$ are the unit vector along $\mathrm{O} \eta_{1}$-axis and the angular velocity of the gyrostat, respectively. The two vectors $\vec{\omega}$ and $\vec{\gamma}$ are referred to the body frame.

The vector $\vec{\gamma}$ is written in terms of Eulerian angle as illustrated in [1]:

$$
\vec{\gamma}=\left(\gamma_{1}, \gamma_{2}, \gamma_{3}\right)=(\sin \theta \sin \varphi, \sin \theta \cos \varphi, \cos \theta),
$$

where $\theta, \psi$, and $\varphi$ indicate the angle of nutation between the two axes $O \eta_{2}$ and $O \eta_{1}$, the precession angle about the $O \eta_{2}$ axis, and the angle of proper rotation, respectively. The homogeneous magnetic field $\overrightarrow{\mathcal{N}}$ is presumed to be a constant and influences in the direction of the $O \eta_{1}$-axis, and therefore, it can be written as

$$
\overrightarrow{\mathcal{N}}=\beta \vec{\gamma},
$$

where $\beta$ denotes the magnitude of the magnetic field. Now, we are going to introduce the equations of the motion. The whole angular momentum of the gyrostat is

$$
\overrightarrow{\mathscr{G}}=\vec{\pi}+\overrightarrow{\mathscr{K}}
$$

where $\vec{\pi}=\square \vec{\omega}$ is the angular momentum of the gyrostat when the rotor is relatively at rest and $\overrightarrow{\mathscr{K}}=(0,0, k)$ is the gyrostatic momentum, that is, the relative angular momentum of the rotor with respect to the body. Following the theorem of angular momentum about the point $O$, fixed point, we obtain

$$
\frac{\mathrm{d} \overrightarrow{\mathscr{G}}}{\mathrm{d} t}=\overrightarrow{\mathscr{M}}_{0},
$$

where $\overrightarrow{\mathscr{M}}_{0}$ indicates the total torque of the external forces about the fixed point $O$.

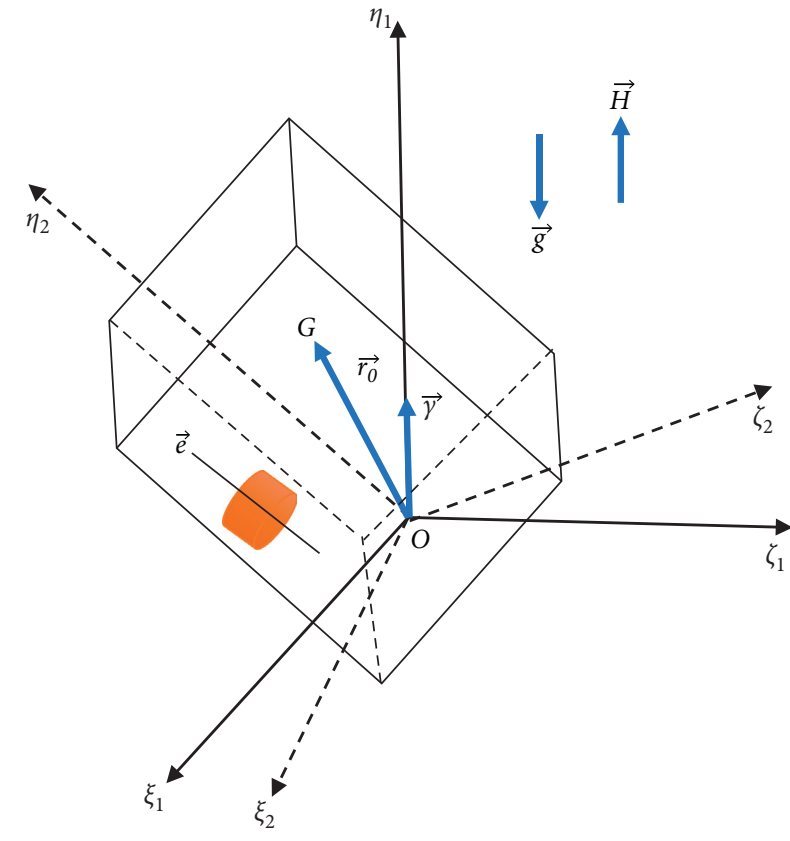

Figure 1: References frames and gyrostat.

According to [1], the potential function for the Newtonian forces field takes the following form:

$$
U\left(\gamma_{1}, \gamma_{2}, \gamma_{3}\right)=m g \overrightarrow{r_{0}} \cdot \vec{\gamma}+\frac{n}{2} \vec{\gamma} \cdot \square \vec{\gamma}
$$

where $\overrightarrow{r_{0}}=\left(x_{0}, y_{0}, z_{0}\right)$ is the center of mass vector, and for simplicity, we assume the center of mass lies on $\mathrm{O} \eta_{2}$ and $n=(3 g / R)$, where $R$ is the distance between the center of the attraction and the fixed point $O(R$ is assumed very large compared with the dimensions of the body). The torque due to the potential forces derived from the potential function (5) is given as follows [24]:

$$
\overrightarrow{\mathscr{M}}_{0}^{1}=\vec{\gamma} \times \frac{\partial U}{\partial \vec{\gamma}}=m g \vec{\gamma} \times \overrightarrow{r_{0}}+n \vec{\gamma} \times \square \vec{\gamma}
$$

Now, we calculate the torque appearing due to the magnetic field about the fixed point $O$. Let $p$ be any point from the body which moves with velocity $\vec{u}(p)$, carry a charge $\mathrm{d} q$, and its position vector $\vec{r}$ with respect to the fixed point $O$. This point is influenced by Lorentz forces $\mathrm{d} \vec{F}=$ $\mathrm{d} q(\vec{u} \times \overrightarrow{\mathscr{H}})=\beta \rho \mathrm{d} V[(\vec{\omega} \times \vec{r}) \times \vec{\gamma}]=\beta \rho \mathrm{d} V[(\vec{\omega} \cdot \vec{\gamma}) \vec{r}-$ $(\vec{r} \cdot \vec{\gamma}) \vec{\omega}]$, where $\rho$ is the charge density and $\mathrm{d} V$ is the element's volume from the body. Hence, the torque arising due to the magnetic field takes the following form:

$$
\overrightarrow{\mathscr{M}}_{0}^{2}=\int_{V} \vec{r} \times \mathrm{d} \vec{F}=\vec{\omega} \times \beta \rho \int_{V} \vec{r}(\vec{r} \cdot \vec{\gamma}) \mathrm{d} V=\vec{\omega} \times A \vec{\gamma},
$$

where $A$ is the $3 \times 3$ constant matrix which is assumed to be $A=\operatorname{diag}(a, b, d)$ for simplicity. Thus, the total torque about the fixed point $O$ is 


$$
\begin{aligned}
\overrightarrow{\mathscr{M}}_{0} & =\overrightarrow{\mathscr{M}}_{0}^{1}+\overrightarrow{\mathscr{M}}_{0}^{2} \\
& =m g \vec{\gamma} \times \vec{r}_{0}+n \vec{\gamma} \times \square \vec{\gamma}+\vec{\omega} \times A \vec{\gamma} .
\end{aligned}
$$

Taking into account the two equations (4) and (8), the equations of motion in the body frame take the following form:

$$
\dot{\vec{\pi}}=-\vec{\omega} \times(\vec{\pi}+\vec{\mu})+m g \vec{\gamma} \times \vec{r}_{0}+n \vec{\gamma} \times \square \vec{\gamma},
$$

where

$$
\vec{\mu}=\overrightarrow{\mathscr{K}}+A \vec{\gamma} .
$$

Notably, the expression (10) represents the torque of the gyroscopic forces (forces rely on the velocity). Because $\vec{\gamma}$ is a unit vector fixed in the space, we obtain

$$
\dot{\vec{\gamma}}=\vec{\gamma} \times \vec{\omega}
$$

Despite the variables utilized in two equations (9) and (11) are not canonical, we can describe this motion by means of a Hamiltonian function in the framework of Lie-Poisson systems. The Hamiltonian function takes the following form:

$$
H=\frac{1}{2}\left(\frac{\pi_{1}^{2}}{A}+\frac{\pi_{2}^{2}}{B}+\frac{\pi_{3}^{2}}{C}\right)+m g z_{0} \gamma_{3}+\frac{n}{2}\left(A \gamma_{1}^{2}+B \gamma_{2}^{2}+C \gamma_{3}^{2}\right) .
$$

According to [25], we write the equations of the motion (9) and (11) as a Hamiltonian-Poisson system that is spanned by the matrix $\Pi_{\vec{\mu}}$ :

$$
\Pi_{\vec{\mu}}=\left(\begin{array}{cccccc}
0 & -\pi_{3}-\mu_{3} & \pi_{2}+\mu_{2} & 0 & -\gamma_{3} & \gamma_{2} \\
\pi_{3}+\mu_{3} & 0 & -\pi_{1}-\mu_{1} & \gamma_{3} & 0 & -\gamma_{1} \\
-\pi_{2}-\mu_{2} & \pi_{1}+\mu_{1} & 0 & -\gamma_{2} & \gamma_{1} & 0 \\
0 & -\gamma_{3} & \gamma_{2} & 0 & 0 & 0 \\
\gamma_{3} & 0 & -\gamma_{1} & 0 & 0 & 0 \\
-\gamma_{2} & \gamma_{1} & 0 & 0 & 0 & 0
\end{array}\right),
$$

as long as the Jacobi identity is verified, i.e.,

$$
\begin{aligned}
\Pi_{\vec{\mu}}^{l i} \partial_{l} \Pi_{\vec{\mu}}^{j k}+\Pi_{\vec{\mu}}^{l j} \partial_{l} \Pi_{\vec{\mu}}^{k i}+\prod_{\vec{\mu}}^{l k} \partial_{l} \Pi_{\vec{\mu}}^{i j}=0, \\
i, j, k=1,2, \ldots, 6 .
\end{aligned}
$$

Alternatively, the condition (14) is equivalently verified if the equation

$$
\vec{\gamma} \cdot \nabla_{\vec{\gamma}} \times \vec{\mu}=0,
$$

is satisfied. By direct calculations, we can prove that vector $\vec{\mu}$, shown in (10), satisfied the condition (15), and consequently, the equations of motion (9) and (11) are rewritten as

$$
\dot{\vec{Y}}=\Pi_{\vec{\mu}} \vec{\nabla} H,
$$

where $Y=\left(\pi_{1}, \pi_{2}, \pi_{3}, \gamma_{1}, \gamma_{2}, \gamma_{3}\right)$ and $\vec{\nabla} H$ is the naive gradient of $H$. In addition to the Hamilton (12), this system admits two Casimirs:

where $\alpha_{0}$ is a constant.

$$
\begin{aligned}
& C_{1}:=\gamma_{1}^{2}+\gamma_{2}^{2}+\gamma_{3}^{2}=1, \quad \text { geometric integral, } \\
& \begin{aligned}
C_{2}:=\pi_{1} \gamma_{1}+\pi_{2} \gamma_{2}+\pi_{3} \gamma_{3}+\frac{1}{2}\left(a \gamma_{1}^{2}+b \gamma_{2}^{2}+d \gamma_{3}^{2}\right)+k \gamma_{3}=\alpha_{0}, \quad \text { area integral, } \\
\quad \gamma_{1}\left[\gamma_{3}(C-A)\left(\omega^{2}-n\right)+\omega\left(k+\gamma_{3}(d-a)\right)-m g z_{0}\right]=0
\end{aligned}
\end{aligned}
$$

\section{Permanent Rotations}

To find the permanent rotations, we place $\dot{\vec{\pi}}=\dot{\vec{\gamma}}=\overrightarrow{0}$ into the two equations of motion (9) and (11); we obtain

$$
\begin{gathered}
\vec{\omega} \times(\vec{\pi}+\overrightarrow{\mathscr{K}}+A \vec{\gamma})-m g \vec{\gamma} \times \vec{r}_{0}-n \vec{\gamma} \times \square \vec{\gamma}=0, \\
\vec{\gamma} \times \vec{\omega}=0 .
\end{gathered}
$$

Equation (19) implies the two vectors $\vec{\omega}$ and $\vec{\gamma}$ are parallel, and consequently, $\vec{\omega}=\omega \vec{\gamma}$, where $\omega$ is the magnitude of angular velocity of the gyrostat in the body frame. Thus, equation (18) becomes

$$
\vec{\gamma} \times\left[\left(\omega^{2}-n\right) \square \vec{\gamma}+\omega(\overrightarrow{\mathscr{K}}+A \vec{\gamma})-m g \vec{r}_{0}\right]=0 .
$$

The scalar form for equation (20) is

$$
\gamma_{2}\left[\gamma_{3}(C-B)\left(\omega^{2}-n\right)+\omega\left(k+\gamma_{3}(d-b)\right)-m g z_{0}\right]=0,
$$

$$
\gamma_{1} \gamma_{2}\left[(B-A)\left(\omega^{2}-n\right)+\omega(b-a)\right]=0
$$

From equation (23), we have three possibilities $\gamma_{1}=\gamma_{2}=0, \gamma_{1}=0, \gamma_{2} \neq 0$, and $\gamma_{1} \neq 0, \gamma_{2}=0$. We study these cases one by one. Hereinafter, the permanent rotation is written in the form $\mathscr{E}=\left(A \omega \gamma_{10}, B \omega \gamma_{20}, C \omega \gamma_{30}, \gamma_{10}, \gamma_{20}, \gamma_{30}\right)$.

(i) When $\gamma_{1}=\gamma_{2}=0$, equations (21)-(23) are satisfied, identically. Using the geometric integral (2), we get $\gamma_{3}= \pm 1$. The permanent rotation is $\mathscr{E}_{1}^{ \pm}=(0,0, \pm C \omega, 0,0, \pm 1) . \mathscr{E}_{1}^{+}$characterizes the rotation of the gyrostat about the vertical axis in the upward direction. This means the angle $\theta$ between the two axes $O \eta_{1}$ and $O \eta_{2}$ is zero; i.e., the fixed point 
$O$ lies down the center of the mass of the gyrostat. In a similitude way, the permanent rotation $\mathscr{E}_{1}^{-}$is explained as the rotation of the gyrostat in the down direction; i.e., the fixed point $O$ lies above the center of mass of the gyrostat.

(ii) When $\gamma_{1}=0$ and $\gamma_{2} \neq 0$, the geometric integral (2) reduces to $\gamma_{2}^{2}+\gamma_{3}^{2}=1$, which has a parametric solution $\gamma_{2}=\sin \theta$ and $\gamma_{3}=\cos \theta$. Taking into account the obtained results, equations (21)-(23) become

$\left[(B-C)\left(n-\omega^{2}\right)+(d-b) \omega+C n\right] \cos \theta+k \omega-m g z_{0}=0$,

which represents the existence condition for a family of the permanent rotations taking the form $\mathscr{E}_{2}=(0, B \omega \sin \theta, C \omega \cos \theta, 0, \sin \theta, \cos \theta)$. It represents a rotation of a gyrostat with a constant angular velocity about an axis having a direction cosine $(0, \sin \theta, \cos \theta)$.

(iii) When $\gamma_{1} \neq 0$ and $\gamma_{2}=0$, the geometric integral becomes $\gamma_{1}^{2}+\gamma_{3}^{2}=1$, which admits the parametric solution $\gamma_{1}=\sin \theta$ and $\gamma_{3}=\cos \theta$. Regarding the obtained results, equations (21)-(23) reduce to

$$
\left[(A-C)\left(n-\omega^{2}\right)+\omega(d-a)\right] \cos \theta+k \omega-m g z_{0}=0
$$

that is, the condition for the existence of the family of the permanent rotations $\mathscr{E}_{3}=(A \omega \sin \theta, 0, C \omega \cos \theta, \sin \theta, 0$, $\cos \theta) . \mathscr{E}_{3}$ is explained as the rotation of a gyrostat with a constant angular velocity about an axis with direction cosines $(\sin \theta, 0, \cos \theta)$.

Collecting the obtained results, we introduce down the following.

Theorem 1. The mechanical system (9) and (11) characterizing the rotations of a charged gyrostat in Newtonian field has four permanent rotations. They are as follows:

(i) $\mathscr{E}_{1}^{ \pm}=(0,0, \pm C \omega, 0,0, \pm 1)$.

(ii) $\mathscr{E}_{2}=(0, B \omega \sin \theta, C \omega \cos \theta, 0, \sin \theta, \cos \theta)$ provided that $\left[(B-C)\left(n-\omega^{2}\right)+(d-b) \omega+C n\right] \cos \theta+k \omega-$ $m g z_{0}=0$ is satisfied.

(iii) $\mathscr{E}_{3}=(A \omega \sin \theta, 0, C \omega \cos \theta, \sin \theta, 0, \cos \theta)$ if the condition $\left[(A-C)\left(n-\omega^{2}\right)+\omega(d-a)\right] \cos \theta+k \omega-$ $m g z_{0}=0$ is verified.

\section{Stability Analysis}

This section aims to examine the stability of the permanent rotations introduced in Theorem 1 . We apply a linear approximation method to determine the sufficient conditions for instability that are also necessary conditions for the stability. We evaluate the tangent flow for the equations of the motion at the permanent rotation $\mathscr{E}$, and we get

$$
\frac{\mathrm{d} \mathbf{y}}{\mathrm{d} t}=\mathfrak{\Im}(\mathrm{E}) \mathbf{y}
$$

where $\mathfrak{J}(\mathscr{E})$ is the Jacobi matrix calculated at the permanent rotation $\mathscr{E}$. To examine the linear stability, we find the eigenvalues of the Jacobi matrix and those eigenvalues are the roots of the characteristic equation

$$
\operatorname{det}\left[\mathfrak{\Im}(\mathscr{E})-\lambda I_{6}\right]=0
$$

where $I_{6}$ refers the $6 \times 6$ identity matrix.

The energy-Casimir method is utilized to find sufficient conditions for stability. This method was employed in several works such as [15-23]. The energy-Casimir method is briefly presented in the following.

Theorem 2. Assuming $(\mathscr{M},\{\cdot, \cdot\}, \mathcal{N})$ is a Poisson system and $\mathscr{E} \in \mathscr{M}$ is an equilibrium point for the Hamiltonian vector $X_{\mathcal{N}}$. If there is a set of Casimirs $C_{i} \in C^{\infty}, i=1,2, \ldots, n$ satisfies

$$
\begin{array}{r}
\mathbf{d}\left(\mathcal{N}+\sum_{i=1}^{n} C_{i}\right)(\epsilon)=0, \\
\left.\mathbf{d}^{2}\left(\mathcal{N}+\sum_{i=1}^{n} C_{i}\right)(\epsilon)\right|_{\mathscr{W} \times \mathscr{W}},
\end{array}
$$

is definite for $\mathscr{W}$ that is defined by

$$
\mathscr{W}=\bigcap_{i=1}^{n} \operatorname{kerd}_{i}(\mathscr{E}) .
$$

Then, $\mathscr{E}$ is stable and $\mathscr{E}$ is usually stable if $\mathscr{W}=\{0\}$.

4.1. Stability of $\mathscr{E}_{1}^{ \pm}$. This section aims to examine the stability of the permanent rotation $\mathscr{E}_{1}^{ \pm}$which describes the rotation about the vertical axis with a constant angular velocity in two cases characterized by whether the fixed point $O$ is above or down the gyrostat center of mass.

To obtain the necessary conditions for the stability, we compute the tangent flow of the equations of the motion (9) and (11) in the permanent rotation $\mathscr{E}_{1}^{ \pm}$, and we obtain an equation in the form (26) and its characteristic equation (27) admits the following form:

$$
\lambda^{2}\left(\lambda^{4}+P_{1} \lambda^{2}+Q_{1}\right)=0
$$

where 


$$
\begin{aligned}
P_{1}= & \frac{1}{A B}\left[(A(A-C)+B(B-C)) n+(C(C-A)+B(2 A-C)) \omega^{2}-m g z_{0}(A+B)+(d+k)^{2}\right. \\
& \mp \omega[A a+B b+(d+k)(A+B-2 C)]] \\
Q_{1}= & \frac{1}{A B}\left[ \pm(B-C)\left(\omega^{2}-n\right)+(b-d-k) \omega \pm m g z_{0}\right]\left[ \pm(A-C)\left(\omega^{2}-n\right)+(a-d-k) \omega \pm m g z_{0}\right] .
\end{aligned}
$$

Thus, we can formulate the following.

Theorem 3. Let a charged gyrostat move about its fixed point $\mathrm{O}$ due to the Newtonian force field, then the necessary condition for its rotation about the vertical axis up or down is linearly stable if $P_{1} \geq 0, Q_{1} \geq 0$, and $P_{1}^{2}-4 Q_{1} \geq 0$. Or, equivalently, this motion is Lyapunov unstable if at least one of these conditions is not satisfied.

Now, we are going to determine the sufficient conditions for the stability by employing the energy-Casimir method that is presented in Theorem 2. We introduce the augmented Hamilton in the following form:

$$
\begin{aligned}
\mathcal{N}= & \frac{1}{2}\left(\frac{\pi_{1}^{2}}{A}+\frac{\pi_{2}^{2}}{B}+\frac{\pi_{3}^{2}}{C}\right)+m g z_{0} \gamma_{3}+\frac{n}{2}\left(A \gamma_{1}^{2}+B \gamma_{2}^{2}+C \gamma_{3}^{2}\right)+v_{1}\left[\gamma_{1}^{2}+\gamma_{2}^{2}+\gamma_{3}^{2}\right] \\
& +v_{2}\left[\pi_{1} \gamma_{1}+\pi_{2} \gamma_{2}+\pi_{3} \gamma_{3}+\frac{1}{2}\left(a \gamma_{1}^{2}+b \gamma_{2}^{2}+d \gamma_{3}^{2}\right)+k \gamma_{3}\right],
\end{aligned}
$$

where $v_{1}$ and $\nu_{2}$ are arbitrary constants which are determined by taking into account and $\mathscr{E}_{1}^{ \pm}$is a critical point for the augmented Hamilton $\mathcal{N}$, i.e.,

$$
\begin{aligned}
\left.\frac{\partial \mathcal{N}}{\partial \pi_{i}}\right|_{\mathscr{E}_{1}^{ \pm}} & =0, \\
\left.\frac{\partial \mathcal{N}}{\partial \gamma_{i}}\right|_{\mathscr{E}_{1}^{ \pm}} & =0, \\
i & =1,2,3 .
\end{aligned}
$$

Equation (33) implies

$$
v_{1}=\frac{C}{2}\left(\omega^{2}-n\right) \pm \frac{1}{2}\left[\omega(k \pm d)-m g z_{0}\right], \quad v_{2}=-\omega .
$$

The subspace $\mathscr{W}$ is determined by

$$
\mathscr{W}=\operatorname{kerd} C_{1}\left(\mathscr{E}_{1}^{ \pm}\right) \cap \operatorname{kerd} C_{2}\left(\mathscr{E}_{1}^{ \pm}\right),
$$

where

$$
\begin{aligned}
& \mathbf{d} C_{1}\left(\mathrm{E}_{1}^{ \pm}\right)= \pm 2 \mathrm{~d} \gamma_{3}, \\
& \mathbf{d} C_{2}\left(\mathrm{E}_{1}^{ \pm}\right)= \pm \mathrm{d} \pi_{3}+(k \pm d \pm C \omega) \mathrm{d} \gamma_{3} .
\end{aligned}
$$

After some calculation, the basis of the subspace $\mathscr{W}$ is

$$
\mathfrak{B}_{\mathscr{W}}=\left\{\vec{e}_{1}, \vec{e}_{2}, \vec{e}_{4}, \vec{e}_{5}\right\}
$$

where $\vec{e}_{i}$ are the canonical basis of $\mathbb{R}^{6}$. The Hessian matrix for the augmented Hamilton (32) in the reduced subspace $\mathscr{W}$ is

$$
\left.\operatorname{Hess}\right|_{\mathscr{W} \times \mathscr{W}}=\left(\begin{array}{cccc}
\frac{1}{A} & 0 & 0 & -\omega \\
0 & \frac{1}{B} & -\omega & 0 \\
0 & -\omega & x & 0 \\
-\omega & 0 & 0 & y
\end{array}\right) \text {, }
$$

where

$$
\begin{aligned}
& x=C \omega^{2}+n(B-C) \mp m g z_{0}+\omega(d \pm k-b), \\
& y=C \omega^{2}+n(A-C) \mp m g z_{0}+\omega(d \pm k-a) .
\end{aligned}
$$

We investigate the definiteness of the Hessian matrix (38) by applying the Sylvester criterion and so we evaluate its principal minors:

$$
\begin{aligned}
& \Delta_{1}=\frac{1}{A}, \\
& \Delta_{2}=\frac{1}{A B} \\
& \Delta_{3}=\frac{1}{A B}\left[(C-B)\left(\omega^{2}-n\right)+\omega(d \pm k-b) \mp m g z_{0}\right] \\
& \Delta_{4}=\Delta_{3}\left[(C-A)\left(\omega^{2}-n\right)+\omega(d \pm k-a) \mp m g z_{0}\right] .
\end{aligned}
$$

It is obvious that $\Delta_{1}>0$ and $\Delta_{2}>0$ while $\Delta_{3}>0$ if 


$$
\pm m g z_{0}<(C-B)\left(\omega^{2}-n\right)+\omega(k+d-b)=\chi_{1},
$$

and $\Delta_{4}>0$ if

$$
\pm m g z_{0}<(C-A)\left(\omega^{2}-n\right)+\omega(k+d-a)=\chi_{2} .
$$

The two inequalities (41) and (42) verify together if

$$
\pm m g z_{0}<\min \left(\chi_{1}, \chi_{2}\right)
$$

Thus, we can formulate the theorem.

Theorem 4. The sufficient condition of the stability for the permanent rotation $\mathscr{E}_{1}^{ \pm}$to be Lyapunov stable is (43).

Figure 2 determines the regions of linear stability and Lyapunov stability for $\mathscr{E}_{1}^{ \pm}$. In Figure 2(a), the regions in pink determine the linear stability, while the white zones represent the instability. Notably, the solid lines in Figure 2 are determined by $P_{1} 0, Q_{1}=0, P_{1}^{2}-4 Q_{1}=0$. Figure $2(\mathrm{~b})$ specifies the regions of Lyapunov stability in yellow, and the dash lines are specified by $m g z_{0}=\min \left(\chi_{1}, \chi_{2}\right)$. Figure $3(\mathrm{c})$ clarifies the regions of Lyapunov stability appears as a portion from the regions of linear stability.

Similar figures can be used to describe the zones of the stability and instability for the permanent rotation $\mathscr{E}_{1}^{-}$.

4.2. Stability of $\mathscr{E}_{2}$. We endeavor to find the necessary and sufficient conditions for a family of the permanent rotation $\mathscr{E}_{2}$ by utilizing the linear approximation and energy-Casimir method, respectively.

Calculating the tangent flow of the equations of the motion (9) and (11) in the equilibrium position $\mathscr{E}_{2}$, we get an equation in the form (26) and its characteristic equation (27) takes the following form:

$$
\lambda^{2}\left(\lambda^{4}+P_{2} \lambda^{2}+Q_{2}\right)=0
$$

where

$$
\begin{aligned}
P_{2}= & \frac{\omega(2 b B-A a) \sin ^{2} \theta}{A C}+\frac{(A+C)}{A C}\left\{\left[(B-A) n+(B-C) \omega^{2}+b \omega\right] \cos ^{2} \theta-B\left(\omega^{2}+n\right)-b \omega\right\} \\
& +\frac{2 k \cos \theta}{A B} \times[d-(A+B-C) \omega] \\
& -\frac{\cos ^{2} \theta}{A B C}\left\{(B-C)\left(B^{2}+C^{2}-2 A C\right) \omega^{2}+C \omega[\mathrm{d} B-2(C-A) d+A(d-a)]-C d^{2}+B b^{2}-n C\left(A^{2}-B C\right)\right\} \\
& +\frac{k^{2}}{A B}+\frac{1}{A C}\left[\omega^{2}\left(B^{2}+2 A C\right)+b^{2}+n\left(A^{2}+C^{2}\right)\right]-\frac{\mathrm{d} \omega}{A}, \\
Q_{2}= & \frac{\sin ^{2} \theta}{A B C}\left[(B-A)\left(\omega^{2}-n\right)-\omega(a-b)\right]\left[\left((B-C)\left(\left(n+3 \omega^{2}\right)(B-C)+3 \omega(b-d)\right)+(b-d)^{2}\right) \cos ^{2} \theta\right. \\
& \left.+2(2 k(B-C) \omega+b-d) \cos \theta+\left(\left(n-\omega^{2}\right)(C-B)-\omega(d-b)\right) B+k^{2}\right] .
\end{aligned}
$$

Notice that we use the existence condition (24) to eliminate the weight of the gyrostat $\mathrm{mg}$. Now, we can write down the following.

Theorem 5. The family of permanent rotation $\mathscr{E}_{2}$ is linearly stable if the conditions $P_{2} \geq 0, Q_{2} \geq 0$, and $P_{2}^{2}-4 Q_{2} \geq 0$ are met. Or, equivalently, it is Lyapunov instable if at least one of these conditions is not satisfied.

To complete our study about the stability of $\mathscr{E}_{2}$, we apply the energy-Casimir method to find the stability's sufficient conditions. We utilize the same augmented Hamilton (34) and determine the values the two constants $\nu_{1}$ and $v_{2}$ leading to $\mathscr{E}_{2}$ becomes a critical point for this augmented Hamilton, i.e.,

$$
\begin{aligned}
& \left.\frac{\partial \mathcal{N}}{\partial \pi_{i}}\right|_{\mathscr{E}_{2}}=0, \\
& \left.\frac{\partial \mathscr{L}}{\partial \gamma_{i}}\right|_{\mathscr{E}_{2}}=0,
\end{aligned}
$$

$$
i=1,2,3 \text {. }
$$

Equation (46) leads to

$$
\begin{aligned}
& \nu_{1}=\frac{1}{2}\left[B\left(\omega^{2}-n\right)+b \omega\right], \\
& \nu_{2}=-\omega .
\end{aligned}
$$

Following Theorem 2, the subspace $\mathscr{W}$ is specified by

$$
\mathscr{W}=\operatorname{kerd} C_{1}\left(\mathscr{E}_{2}\right) \cap \operatorname{kerd} C_{2}\left(\mathscr{E}_{2}\right),
$$




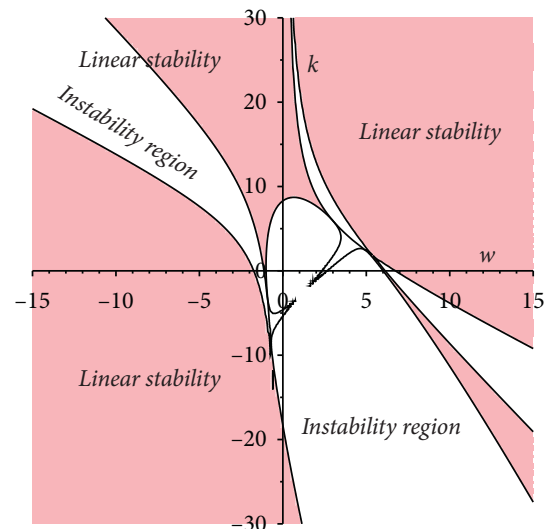

(a)

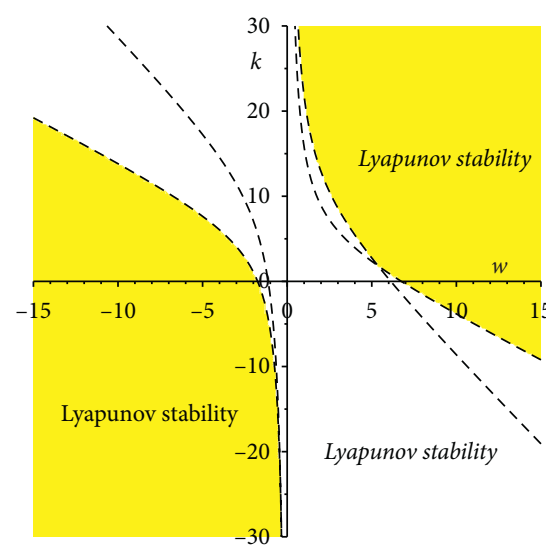

(b)

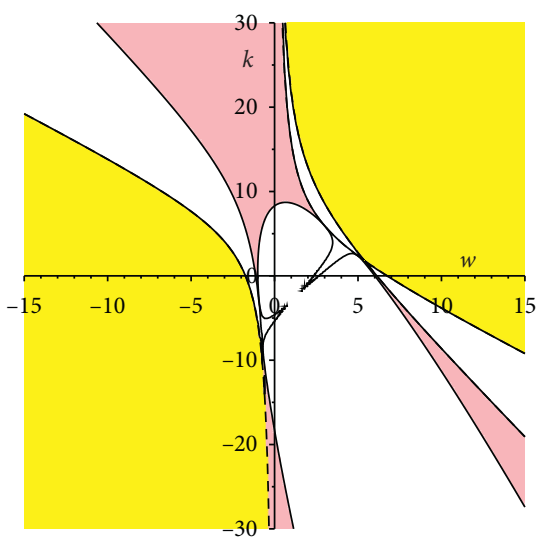

(c)

FIGURE 2: Regions of stability and instability for the permanent rotation $\mathscr{E}_{1}^{+}$in the plane of the two parameters $k$ and $\omega$ and $A=3, B=4, C=5, d=5, b=10, a=15, g=9.8, m=1, z 0=1$.

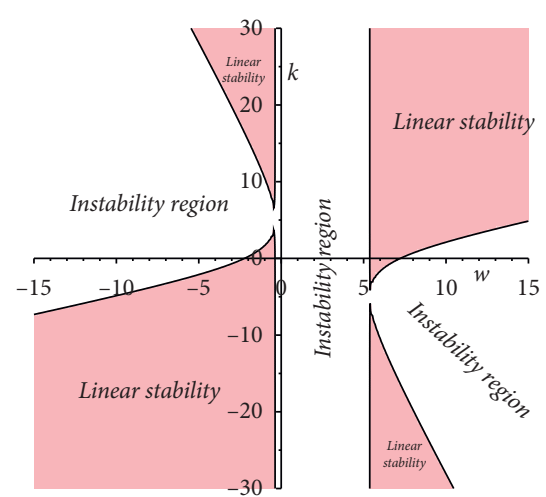

(a)

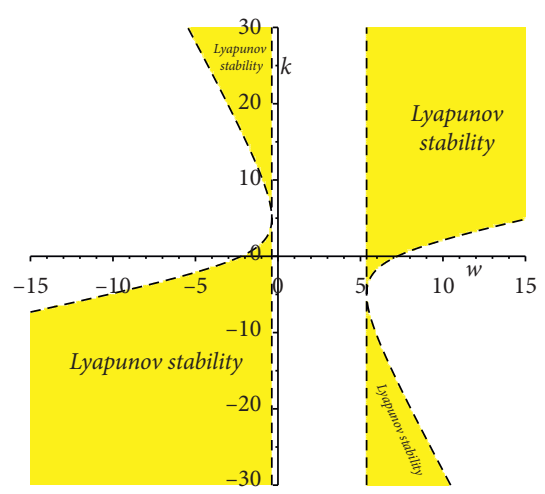

(b)

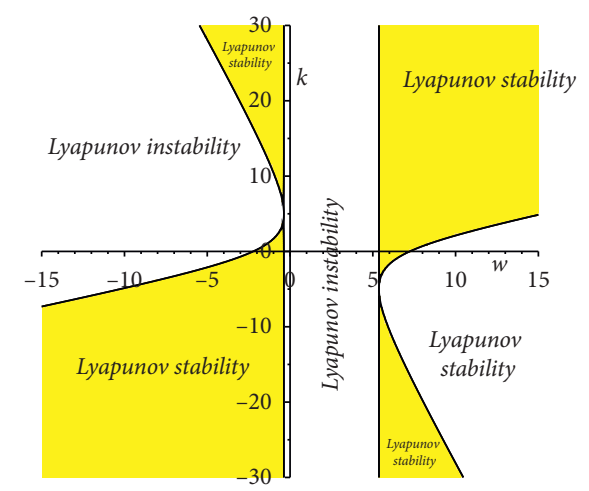

(c)

Figure 3: Regions of stability and instability for the permanent rotation $\mathscr{E}_{2}$ in the plane of the two parameters $k$ and $\omega$ and $A=3, B=4, C=5, d=5, b=10, a=15, g=9.8, m=1, z 0=1$.

where

$$
\begin{aligned}
& \mathbf{d} C_{1}\left(\mathscr{E}_{2}\right)=\mathrm{d} \gamma_{3}+\frac{\sin \theta}{\cos \theta} \mathrm{d} \gamma_{2}, \\
& \mathbf{d} C_{2}\left(\mathscr{E}_{2}\right)=\frac{\sin \theta}{\cos \theta} \mathrm{d} \pi_{2}+\mathrm{d} \pi_{3}+\frac{\sin \theta}{\cos ^{2} \theta}[((B-C) \omega+b-d) \cos \theta-k] \mathrm{d} \gamma_{2} .
\end{aligned}
$$

After some manipulations, we show that the subspace $\mathscr{W}$ is spanned by the vectors

$$
\vec{e}_{1}, \cos \theta \vec{e}_{2}-\sin \theta \vec{e}_{3}, \vec{e}_{4}, \sin \theta[\cos (\theta)((B-C) \omega+b-d)-k] \vec{e}_{3}-\cos ^{2} \theta \vec{e}_{5}+\sin \theta \cos \theta \vec{e}_{6}
$$


where $\vec{e}_{i}$ are the canonical basis of $\mathbb{R}^{6}$. The Hessian matrix corresponding to the Hamiltonian (32) with (47) takes the following form:

$$
\left.\operatorname{Hess}\right|_{\mathscr{W} \times \mathscr{W}}=\left(\begin{array}{cccc}
\frac{1}{A} & 0 & 0 & -\omega \\
0 & \frac{\cos ^{2} \theta}{B}+\frac{\sin ^{2} \theta}{C} \delta_{1} & 0 \\
0 & \delta_{1} & \delta_{2} & 0 \\
-\omega & 0 & 0 & B \omega^{2}+(b-a) \omega+n(A-B)
\end{array}\right) \text {, }
$$

where

$$
\begin{aligned}
\delta_{1}= & \frac{1}{C}\left[((B-C) \omega+b-d) \cos ^{3} \theta-k \cos ^{2} \theta+((2 C-B) \omega-b+d) \cos \theta+k\right], \\
\delta_{2}= & B \omega^{2} \cos ^{4} \theta+\frac{\sin ^{2} \theta \cos ^{2} \theta}{C}\left[\omega^{2}\left(B^{2}-3 B C+3 C^{2}\right)+(b-d)(2 B-3 C) \omega+C n(C-B)+(b-d)^{2}\right] \\
& -\frac{2 k \sin ^{2} \theta \cos \theta}{C}[(B-2 C) \omega+b-d]+k^{2} \sin ^{2} \theta .
\end{aligned}
$$

To investigate the definiteness of Hessian matrix (51), we utilize the Sylvester criterion and so we compute the principal minors. They take the following form:

$$
\begin{aligned}
\Delta_{1}= & \frac{1}{A} \\
\Delta_{2}= & \frac{1}{A}\left[\frac{\cos ^{2} \theta}{B}+\frac{\sin ^{2} \theta}{C}\right], \\
\Delta_{3}= & \frac{\sin ^{2} \theta \cos ^{2} \theta}{A B C}\left\{\left[(B-C)^{2}\left(3 \omega^{2}+n\right)+3 \omega(b-d)(B-C)-(b-d)^{2}\right] \cos ^{2} \theta-2 k[2 \omega(B-C)+b-d] \cos \theta\right. \\
& \left.+B(B-C)\left(\omega^{2}-n\right)+B \omega(b-d)-k^{2}\right\}, \\
\Delta_{4}= & {\left[(B-A)\left(\omega^{2}-n\right)+(b-a) \omega\right] \Delta_{3} . }
\end{aligned}
$$

It is clear that $\Delta_{1}$ and $\Delta_{2}$ are always positive, whereas $\Delta_{3}$ and $\Delta_{4}$ are positive if

$$
\begin{aligned}
& \frac{\sin ^{2} \theta \cos ^{2} \theta}{A B C}\left\{\left[(B-C)^{2}\left(3 \omega^{2}+n\right)+3 \omega(b-d)(B-C)-(b-d)^{2}\right] \cos ^{2} \theta\right. \\
& \left.-2 k[2 \omega(B-C)+b-d] \cos \theta+B(B-C)\left(\omega^{2}-n\right)+B \omega(b-d)-k^{2}\right\}>0, \\
& \Phi(\omega):=(B-A)\left(\omega^{2}-n\right)+(b-a) \omega>0 .
\end{aligned}
$$


$\Phi(\omega)$ is a quadratic polynomial in $\omega$, and its discriminant is always positive and so it has two real roots (say) $\omega_{1}, \omega_{2}$. Thus, we have two cases: (1) when $B>A$, the condition (55) is satisfied if $\omega \in]-\infty, \omega_{1}[\cup] \omega_{2}, \infty[$; (2) if $B<A$, the condition (55) is verified if $\omega \in] \omega_{1}, \omega_{2}[$. Notice that if $A=B$ and $b=a$, the energy-Casimir does not furnish any information about the sufficient conditions of the stability.

Theorem 6. The sufficient condition for the stability of the equilibrium position $\mathscr{E}_{2}$ is

$$
\begin{aligned}
& {\left[(B-C)^{2}\left(3 \omega^{2}+n\right)+3 \omega(b-d)(B-C)-(b-d)^{2}\right] \cos ^{2} \theta} \\
& \quad-2 k[2 \omega(B-C)+b-d] \cos \theta+B(B-C)\left(\omega^{2}-n\right)+B \omega(b-d)-k^{2}>0,
\end{aligned}
$$

with one of the two conditions $B>A, \omega \in]-\infty, \omega_{1}[\bigcup] \omega_{2}$, $\infty[$ or $B<A, \omega \in] \omega_{1}, \omega_{2}[$ is verified.

The regions of linear and nonlinear (Lyapunov) stability are clarified by Figure 3. Figure 3(a) delimits the regions of linear stability in pink color, while as the white regions display the sufficient conditions for the instability of the permanent rotation $\mathscr{E}_{2}$. Notably, the solid lines which are the boundary of those regions are characterized by the equations $P_{2}=0, Q_{2}=0, P_{2}^{2}-4 Q_{2}=0$. The regions in yellow appear in Figure 3(b) represent the regions of Lyapunov stability for $\mathscr{E}_{2}$. Figure $3($ c) illustrates that the regions of Lyapunov stability appear as a part of the regions of linear stability. Notably, on the boundary of linear stability regions (solid lines in Figure 3(a) and also in Figure 3(c)), the permanent rotation $\mathscr{E}_{2}$ is unstable.

4.3. Stability of $\mathscr{E}_{3}$. The stability analysis for the family of the permanent rotations $\mathscr{E}_{3}$ is done by applying similar procedures to $\mathscr{E}_{2}$ stability study. The necessary and sufficient conditions for the stability of $\mathscr{E}_{3}$ can be obtained by replacing $A \leftrightarrow B$ and $a \leftrightarrow b$ in the Theorems 5 and 6 , respectively. Thus, we can formulate the following two theorems.

Theorem 7. The family of permanent rotations $\mathscr{E}_{3}$ is linearly stable if

$$
\begin{aligned}
P_{3}= & \frac{\omega(2 a A-B b) \sin ^{2} \theta}{B C}+\frac{(B+C)}{B C}\left\{\left[(A-B) n+(A-C) \omega^{2}+a \omega\right] \cos ^{2} \theta-A\left(\omega^{2}+n\right)-a \omega\right\}+\frac{2 k \cos \theta}{A B} \\
& \times[d-(A+B-C) \omega]-\frac{\cos ^{2} \theta}{A B C}\left\{(A-C)\left(A^{2}+C^{2}-2 B C\right) \omega^{2}+C \omega[\mathrm{d} A-2(C-B) d+B(d-b)]\right. \\
& \left.-C d^{2}+A a^{2}-n C\left(B^{2}-A C\right)\right\}+\frac{k^{2}}{A B}+\frac{1}{B C}\left[\omega^{2}\left(A^{2}+2 B C\right)+a^{2}+n\left(B^{2}+C^{2}\right)\right]-\frac{\mathrm{d} \omega}{B} \geq 0, \\
Q_{3}= & \frac{\sin ^{2} \theta}{A B C}\left[(A-B)\left(\omega^{2}-n\right)-\omega(b-a)\right]\left[\left((A-C)\left(\left(n+3 \omega^{2}\right)(A-C)+3 \omega(a-d)\right)+(a-d)^{2}\right) \cos ^{2} \theta\right. \\
& \left.+2(2(A-C) \omega+a-d) k \cos \theta+\left(\left(n-\omega^{2}\right)(C-A)-\omega(d-a)\right) A+k^{2}\right] \geq 0, \\
P_{3}^{2}-4 Q_{3}^{2} \geq & 0 .
\end{aligned}
$$

Or, equivalently, it is unstable if at least one of the conditions (57) is verified.
Theorem 8. The sufficient condition for the stability of the equilibrium position $\mathscr{E}_{3}$ is

$$
\begin{aligned}
& {\left[(A-C)^{2}\left(3 \omega^{2}+n\right)+3 \omega(a-d)(A-C)-(a-d)^{2}\right] \cos ^{2} \theta} \\
& \quad-2 k[2 \omega(A-C)+a-d] \cos \theta+A(A-C)\left(\omega^{2}-n\right)+A \omega(a-d)-k^{2}>0,
\end{aligned}
$$

where one of the two conditions $B>A, \omega \in]-\infty, \omega_{1}[\bigcup] \omega_{1}$, $\infty[$ or $A<B, \omega \in] \omega_{1}, \omega_{2}[$ is verified.

Figure 4 displays the regions of sufficient and necessary conditions for the family of permanent rotations $\mathscr{E}_{3}$.
Figure 4(a) illustrates the regions on linear stability in pink, while the zones of Lyapunov instability delimit in white and the solid lines which separate these zones are determined by $P_{3}=0, Q_{3}=0, P_{3}^{2}-4 Q_{3}=0$. Figure $4(\mathrm{~b})$ determines the region of Lyapunov stability in yellow. Figure 4(c) clarifies 


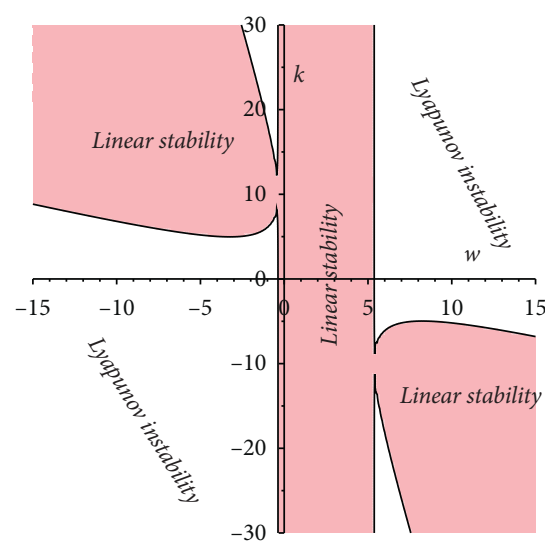

(a)

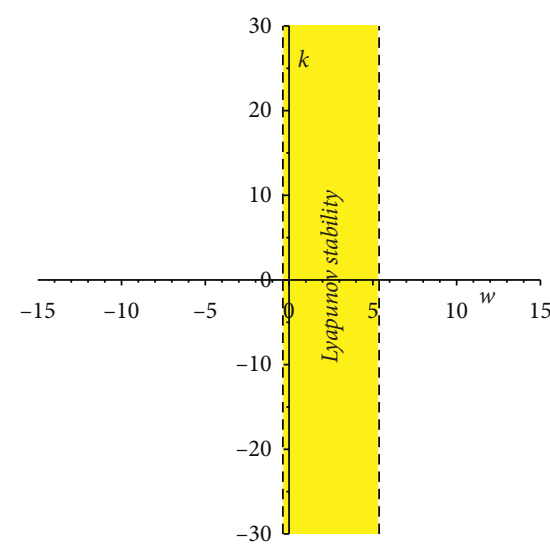

(b)

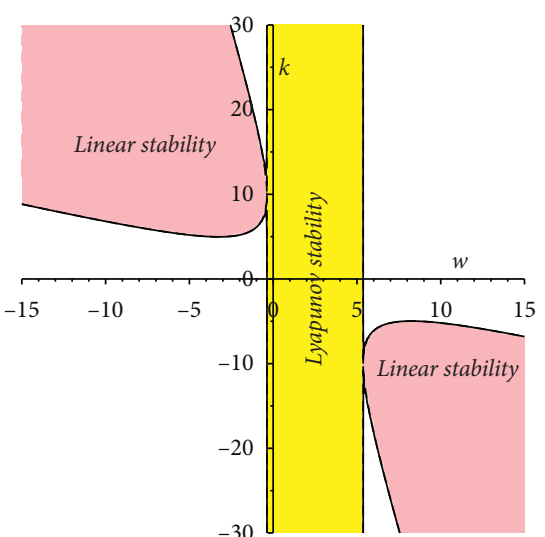

(c)

FIgURE 4: Regions of stability and instability for the permanent rotation $\mathscr{E}_{4}$ in the plane of the two parameters $k$ and $\omega$ and $A=3, B=4, C=5, d=5, b=10, a=15, g=9.8, m=1, z 0=1$.

the zones of Lyapunov stability appear as a portion of linear stability.

\section{Conclusions}

In this work, we have considered the motion of a charged rigid body carrying a rotor rotating about an axis which is parallel to one of the principal axes with a constant velocity. We have assumed the motion happens due to the combination influence of a uniform constant magnetic field and Newtonian force field. The equations of the motion have been constructed and rewritten as a Lie-Poisson Hamilton system. The permanent rotations $\mathscr{E}_{1}$ have been obtained and collected in Theorem 1. The first two permanent rotations $\mathscr{E}_{1}^{ \pm}$are mechanically interpreted as the rotation of the gyrostat about the vertical up or down with a constant angular velocity. The permanent rotation $\mathscr{E}_{2}$ is explained as the rotation of a gyrostat with a constant angular velocity about an axis having a direction $\operatorname{cosine}(0, \sin \theta, \cos \theta)$. The permanent rotation $\mathscr{E}_{3}$ is interpreted as the rotation of the gyrostat with a constant angular velocity about an axis having a direction cosine $(\sin \theta, 0, \cos \theta)$. The linear stability of those equilibrium positions has been studied by applying the linear approximation method, and the obtained results have been collected in Theorems 3, 5, and 7. However, the Lyapunov stability of those permanent rotations have been examined by utilizing the energy-Casimir method, and the finding results have been presented in Theorems 4,6 , and 8 . In the plane of the two variables $k$ and $\omega$, the regions of linear stability have been clarified in pink (see Figures 2(a), 3(a), and 4(a)), while the regions of Lyapunov stability have been illustrated in yellow (see Figures 2(b), 3(b), and 4(b)). Moreover, we illustrate the regions of Lyapunov stability appear as a part of the regions of linear stability.

\section{Data Availability}

No data were used to support the study.

\section{Conflicts of Interest}

The authors declare that they have no conflicts of interest.

\section{References}

[1] E. Leimanis, The General Problem of the Motion of Coupled Rigid Bodies about a Fixed Point, Springer, Berlin, Germany, 1965.

[2] V. Volterra, "Sur la théorie des variations des latitudes," Acta Mathematica, vol. 22, pp. 201-357, 1899.

[3] A. Gluhovsky and C. Tong, "The structure of energy conserving low-order models," Physics of Fluids, vol. 11, no. 2, pp. 334-343, 1999.

[4] P. C. Hughes, Spacecraft Attitude Dynamics, Wiley, New York, NY, USA, 1986.

[5] A. V. Borisov and I. S. Mamaev, Rigid Body DynamicsHamiltonian Methods, Integrability. Chaos. Institute of Computer Science, Izhevsk, Russia, 2001.

[6] H. M. Yehia and A. A. Elmandouh, "New conditional integrable cases of motion of a rigid body with Kovalevskaya's configuration," Journal of Physics A: Mathematical and Theoretical, vol. 44, Article ID 012001, 2011.

[7] H. M. Yehia and A. A. Elmandouh, "A new integrable problem with a quartic integral in the dynamics of a rigid body," Journal of Physics A: Mathematical and Theoretical, vol. 46, no. 14, Article ID 142001, 2013.

[8] A. A. Elmandouh, "New integrable problems in rigid body dynamics with quartic integrals," Acta Mechanica, vol. 226, no. 8, pp. 2461-2472, 2015.

[9] A. A. Elmandouh, "New integrable problems in the dynamics of particle and rigid body," Acta Mechanica, vol. 226, no. 11, pp. 3749-3762, 2015.

[10] H. M. Yehia and A. M. Hussein, "New families of integrable two-dimensional systems with quartic second integrals," Nelineinaya Dinamika, vol. 16, no. 2, pp. 211-242, 2020.

[11] V. Tsogas, T. J. Kalvouridis, and A. G. Mavraganis, "Equilibrium states of a gyrostat satellite in an annular configuration of N big bodies," Acta Mechanica, vol. 175, no. 1-4, pp. 181-195, 2005.

[12] A. Elipe and V. Lanchares, "Two equivalent problems: gyrostats in free motion and parametric quadratic 
Hamiltonians," Mechanics Research Communications, vol. 24, no. 6, pp. 583-590, 1997.

[13] T. Kalvouridis and V. Tsogas, "Rigid body dynamics in the restricted ring problem of $n+1$ bodies," Astrophysics and Space Science, vol. 282, no. 4, pp. 749-763, 2002.

[14] T. S. Amer, A. A. Galal, I. M. Abady, and H. F. Elkafly, "The dynamical motion of a gyrostat for the irrational frequency case," Applied Mathematical Modelling, vol. 89, pp. 12351267, 2020.

[15] T. S. Amer, "On the rotational motion of a gyrostat about a fixed point with mass distribution," Nonlinear Dynamics, vol. 54, no. 3, pp. 189-198, 2008.

[16] J. A. Vera and A. Vigueras, "Hamiltonian dynamics of a gyrostat in the n-body problem: relative equilibria," Celestial Mechanics and Dynamical Astronomy, vol. 94, no. 3, pp. 289-315, 2006.

[17] M. Iñarrea, V. Lanchares, A. I. Pascual, and A. Elipe, "Stability of the permanent rotations of an asymmetric gyrostat in a uniform Newtonian field," Applied Mathematics and Computation, vol. 293, pp. 404-415, 2017.

[18] J. L. G. Guirao and J. A. Vera, "Equilibria, stability and Hamiltonian Hopf bifurcation of a gyrostat in an incompressible ideal fluid," Physica D: Nonlinear Phenomena, vol. 241, no. 19, pp. 1648-1654, 2012.

[19] J. A. Vera, "The gyrostat with a fixed point in a Newtonian force field: relative equilibria and stability," Journal of Mathematical Analysis and Applications, vol. 401, no. 2, pp. 836-849, 2013.

[20] A. A. Elmandouh, "On the stability of the permanent rotations of a charged rigid body-gyrostat," Acta Mechanica, vol. 228, no. 11, pp. 3947-3959, 2017.

[21] A. A. Elmandouh, "On the stability of certain motions of a rigid body-gyrostat in an incompressible ideal fluid," International Journal of Non-linear Mechanics, vol. 120, Article ID 103419, 2020.

[22] A. A. Elmandouh and A. G. Ibrahim, "Hamiltonian structure, equilibria, and stability for an axisymmetric gyrostat motion in the presence of gravity and magnetic fields," Acta Mechanica, vol. 230, no. 7, pp. 2539-2548, 2019.

[23] M. Iñarrea, V. Lanchares, A. I. Pascual, and A. Elipe, "On the stability of a class of permanent rotations of a heavy asymmetric gyrostat," Regular and Chaotic Dynamics, vol. 22, no. 7, pp. 824-839, 2017.

[24] H. M. Yehia, "On the motion of a rigid body acted upon by potential and gyroscopic forces: I. The equations of motion and their transformations," Journal of Mechanical Theory and Applications, vol. 5, pp. 747-754, 1986.

[25] P. Birtea, I. Caşu, and D. Comănescu, "Hamilton-Poisson formulation for the rotational motion of a rigid body in the presence of an axisymmetric force field and a gyroscopic torque," Physics Letters A, vol. 375, no. 45, pp. 3941-3945, 2011. 